\title{
A Thermal Analysis of a Hot-Wire Probe for Icing Applications
}

\author{
Peter Struk \\ NASA GRC \\ Dave Rigby \\ NASA GRC, Vantage \\ Krishna Venkataraman \\ University of Texas, Austin
}




\section{Motivation}

- Further understand behavior of hot-wire measurements in icing conditions

- Newer applications in ice crystals and mixed phase
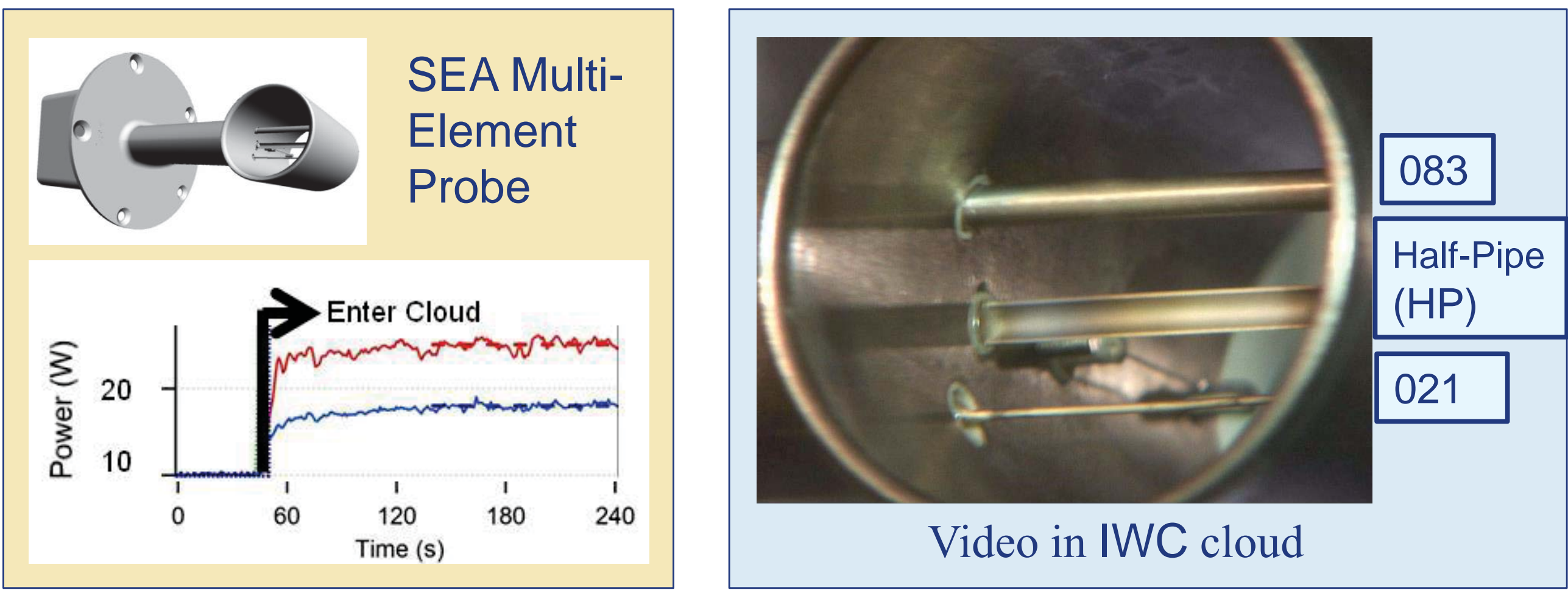

Particle impingement uniformity $\rightarrow$ Rigby et al. (Aviation 2014) Temperature profile along wire $\rightarrow$ Thermal model (this paper) 


\section{Outline}

- Describe Thermal Model

- Governing equations

- Model parameters

- Results (model vs. experiment)

- Dry (air flow only, no water)

- Wet (i.e. cloud on with LWC or IWC)

- Conclusions 


\section{Thermal Model Based on Energy Balance}
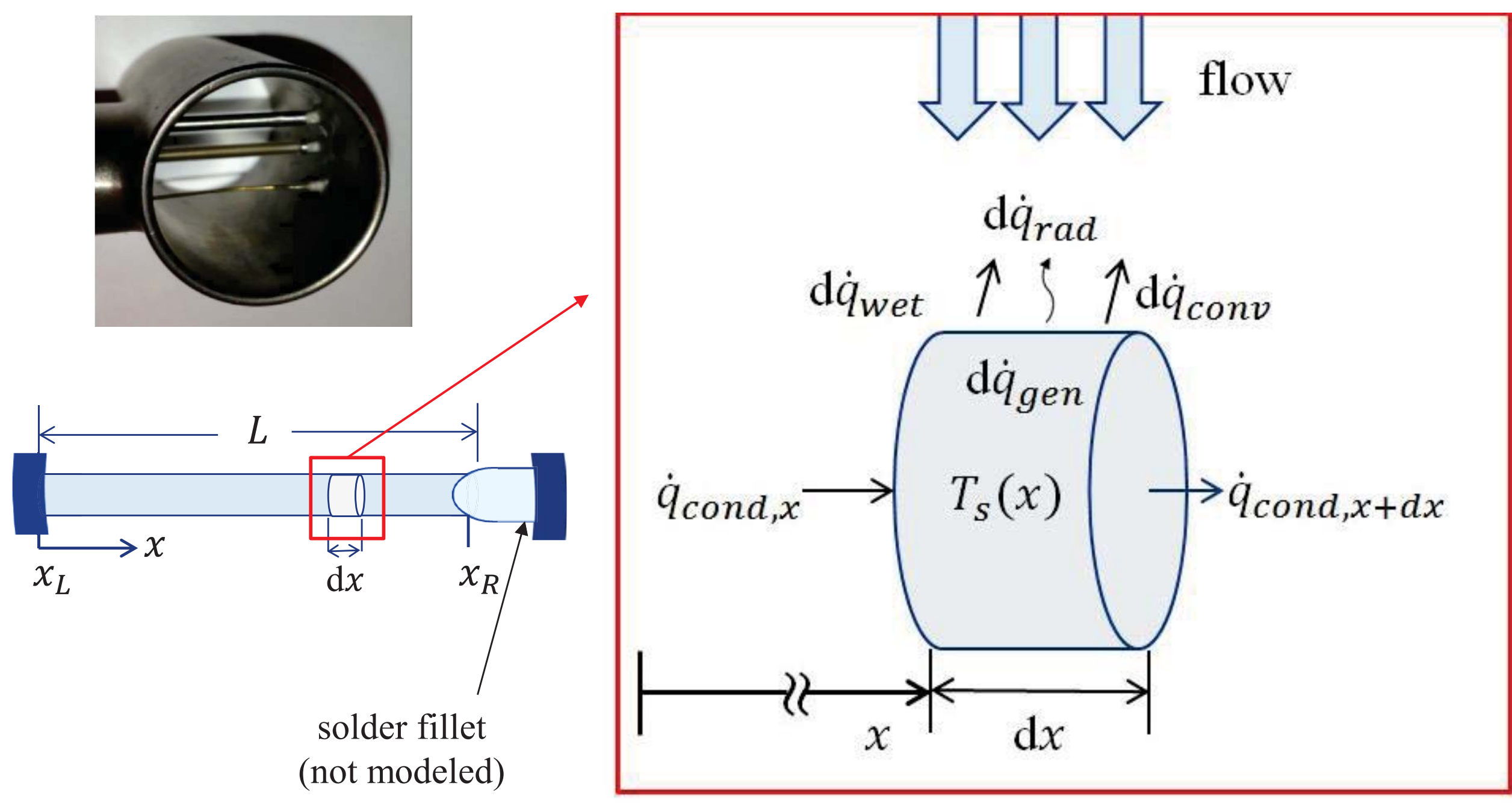

$d \dot{q}_{g e n}=d \dot{q}_{c o n v}+d \dot{q}_{c o n d}+d \dot{q}_{r a d}+d \dot{q}_{w e t}$ 


\section{Governing Equation}

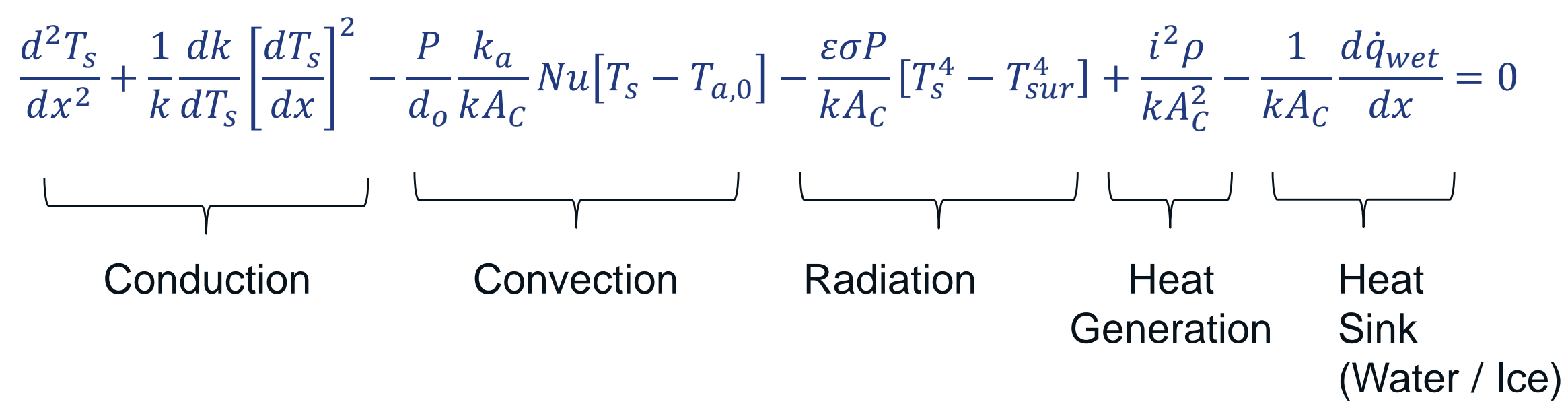
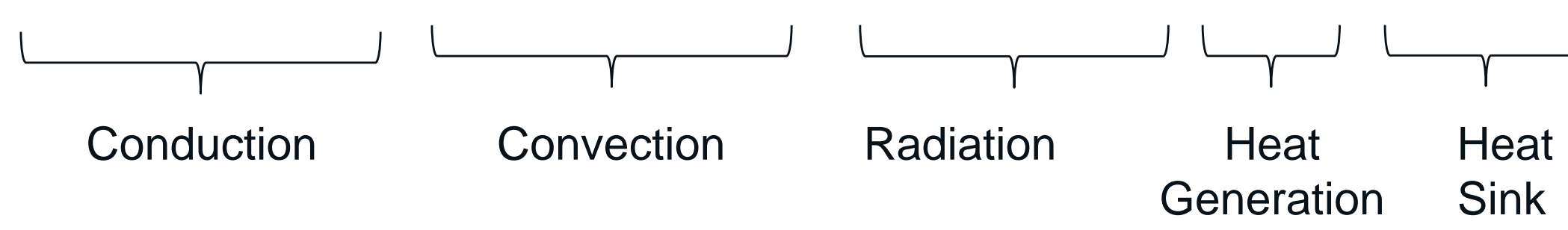

$2^{\text {nd }}$ Order, Non-Linear ODE solved using MATLAB routine bvp4c

Boundary Value Problem need two boundary conditions 


\section{Model Parameters}

- Boundary Conditions
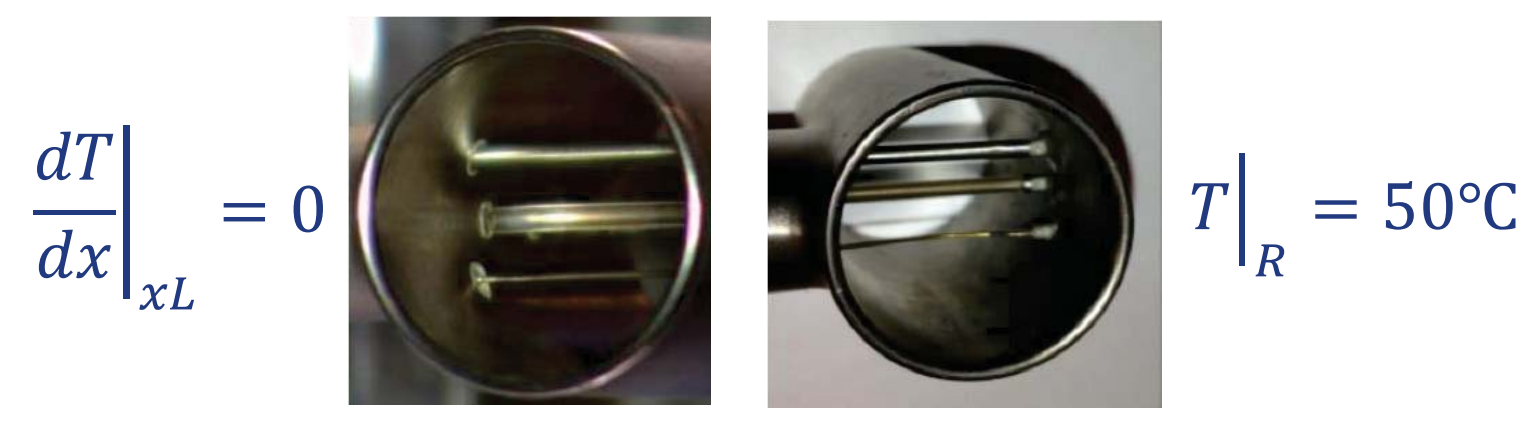

- Convection correlations

- (1) Sparrow et. al - 2004

- (2) Generated from CFD

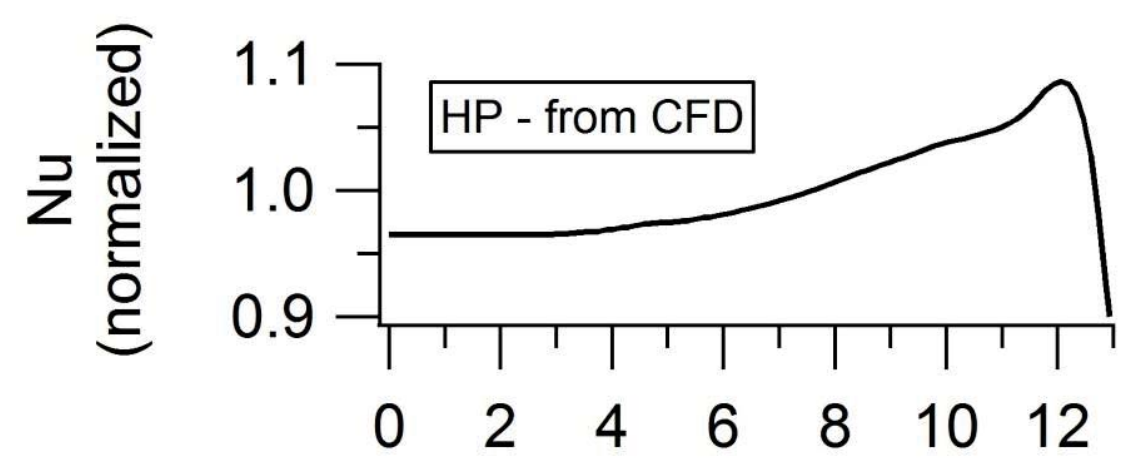

Position from wire center $(\mathrm{mm})$

- Probe operation

- Maintains const. avg. temp (e.g. resistance) $\sim 140{ }^{\circ} \mathrm{C}$

- Temp (Res) $\downarrow$, Power $\uparrow$

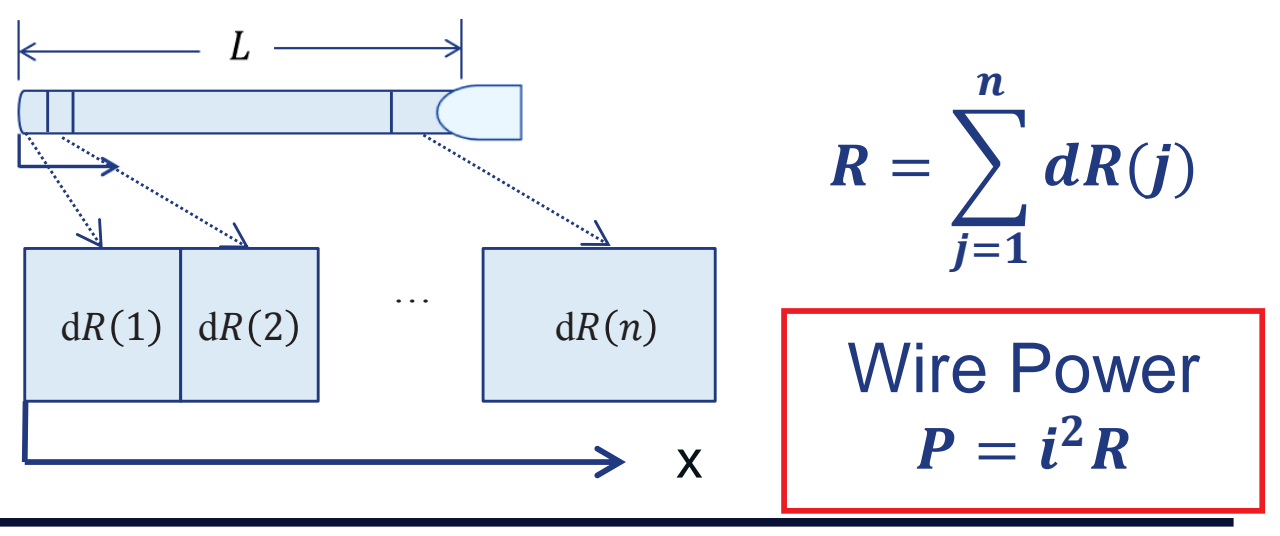




\section{Results}

- Experimental results from 2012 NRC RATFac Tests

- Dry Conditions $\left(P_{0}=13.5,6.5\right.$ psia; $\left.U=85,100,135 \mathrm{~m} / \mathrm{s}\right)$

- Temperature profiles

- Total power (experiment vs. model)

- Effect of heat-transfer coefficient

- Wet results $\left(P_{0}=13.5\right.$ psia, $\left.U=85 \mathrm{~m} / \mathrm{s}\right)$

- LWC Sweep 0-3 g/m ${ }^{3}$

- IWC Sweep 0-10 g/m 


\section{Results: Temperature Profiles - Dry}
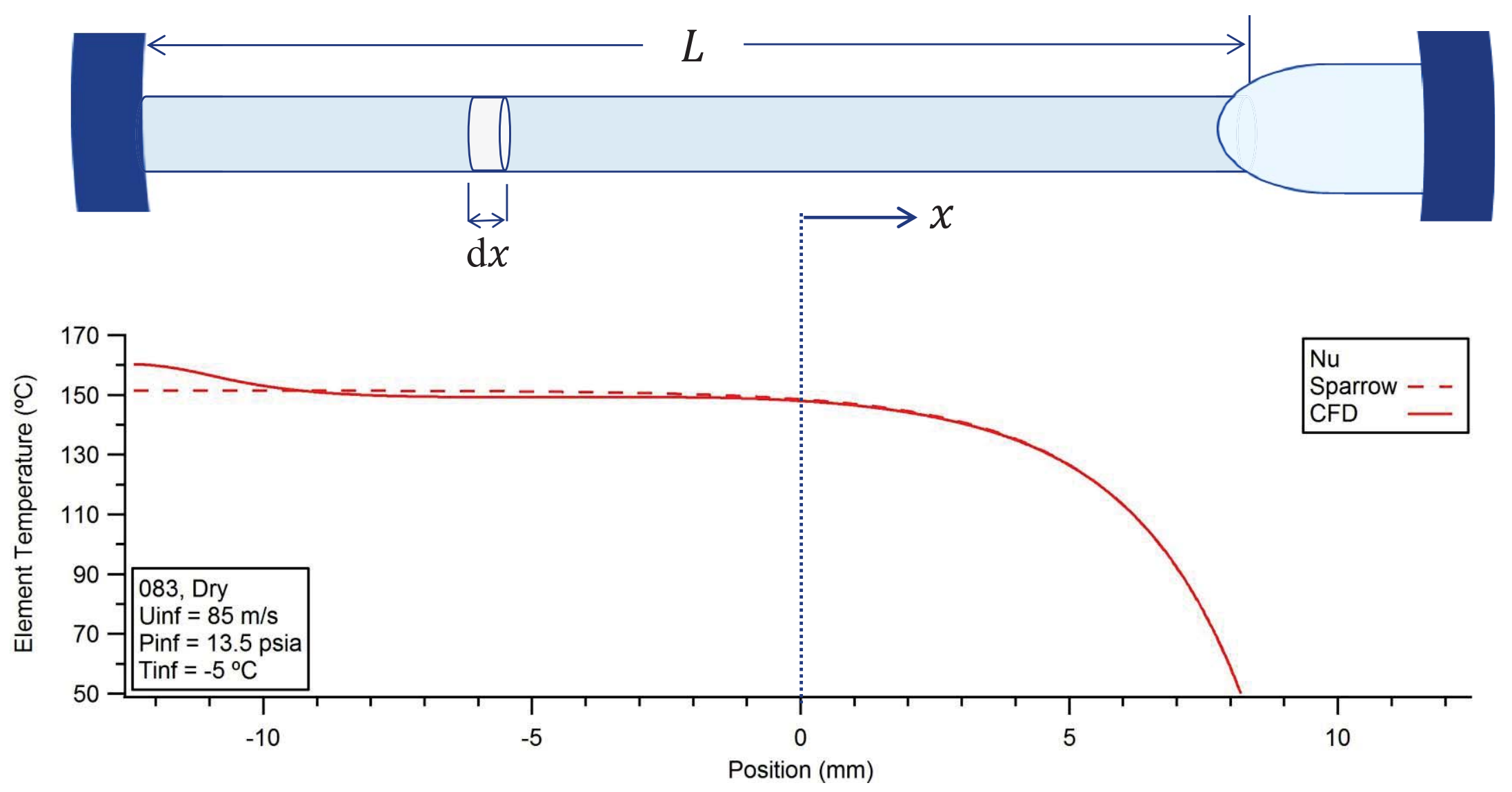


\section{$d q / d x$ along Wire - Dry}
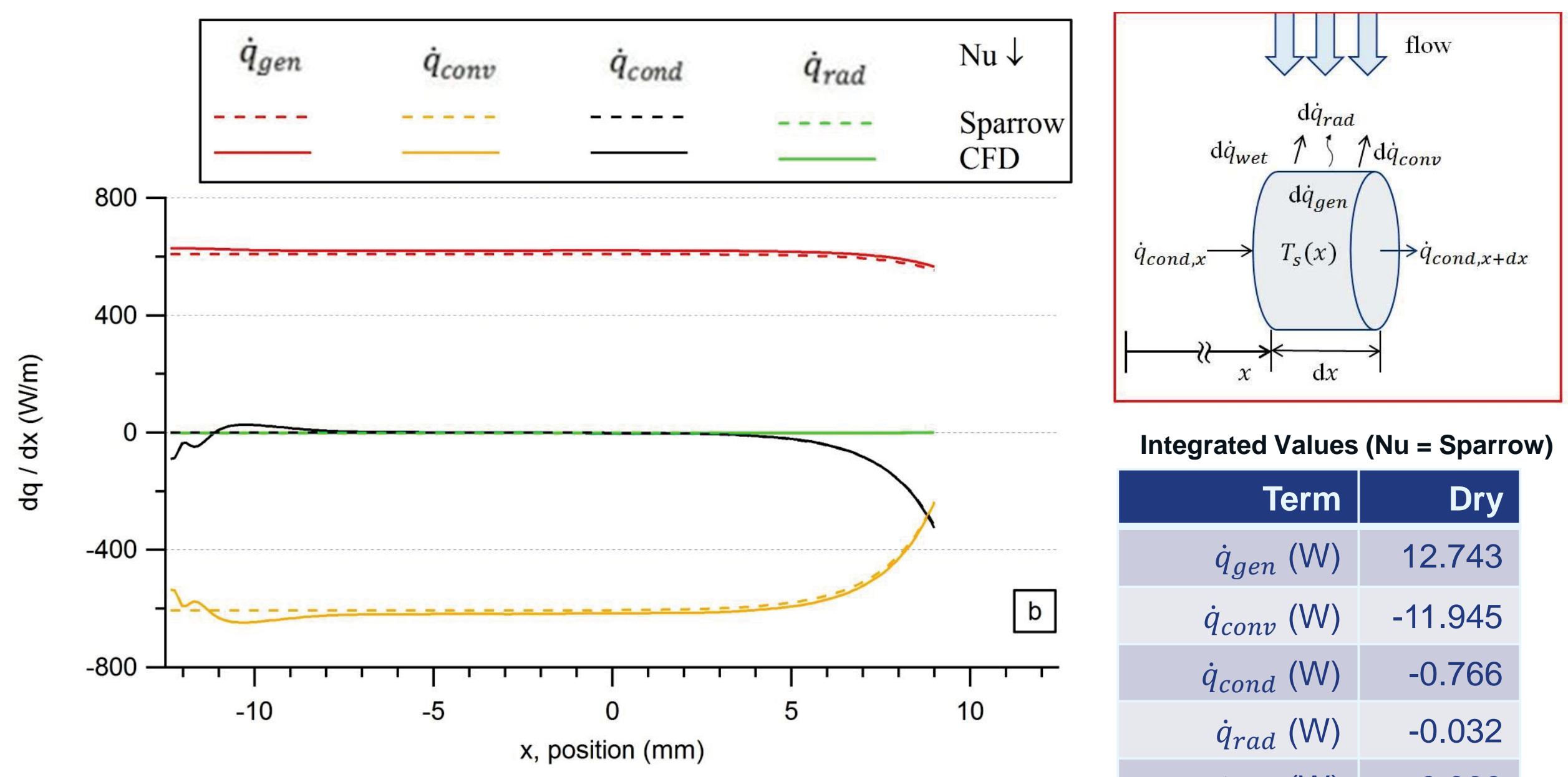

Integrated Values ( $\mathrm{Nu}=$ Sparrow)

\begin{tabular}{|r|r|}
\hline Term & Dry \\
\hline$\dot{q}_{\text {gen }}(\mathrm{W})$ & 12.743 \\
\hline$\dot{q}_{\text {conv }}(\mathrm{W})$ & -11.945 \\
\hline$\dot{q}_{\text {cond }}(\mathrm{W})$ & -0.766 \\
\hline$\dot{q}_{\text {rad }}(\mathrm{W})$ & -0.032 \\
\hline$\dot{q}_{\text {wet }}(\mathrm{W})$ & 0.000 \\
\hline Sum & 0.000 \\
\hline
\end{tabular}




\section{Experimental Data - Dry}

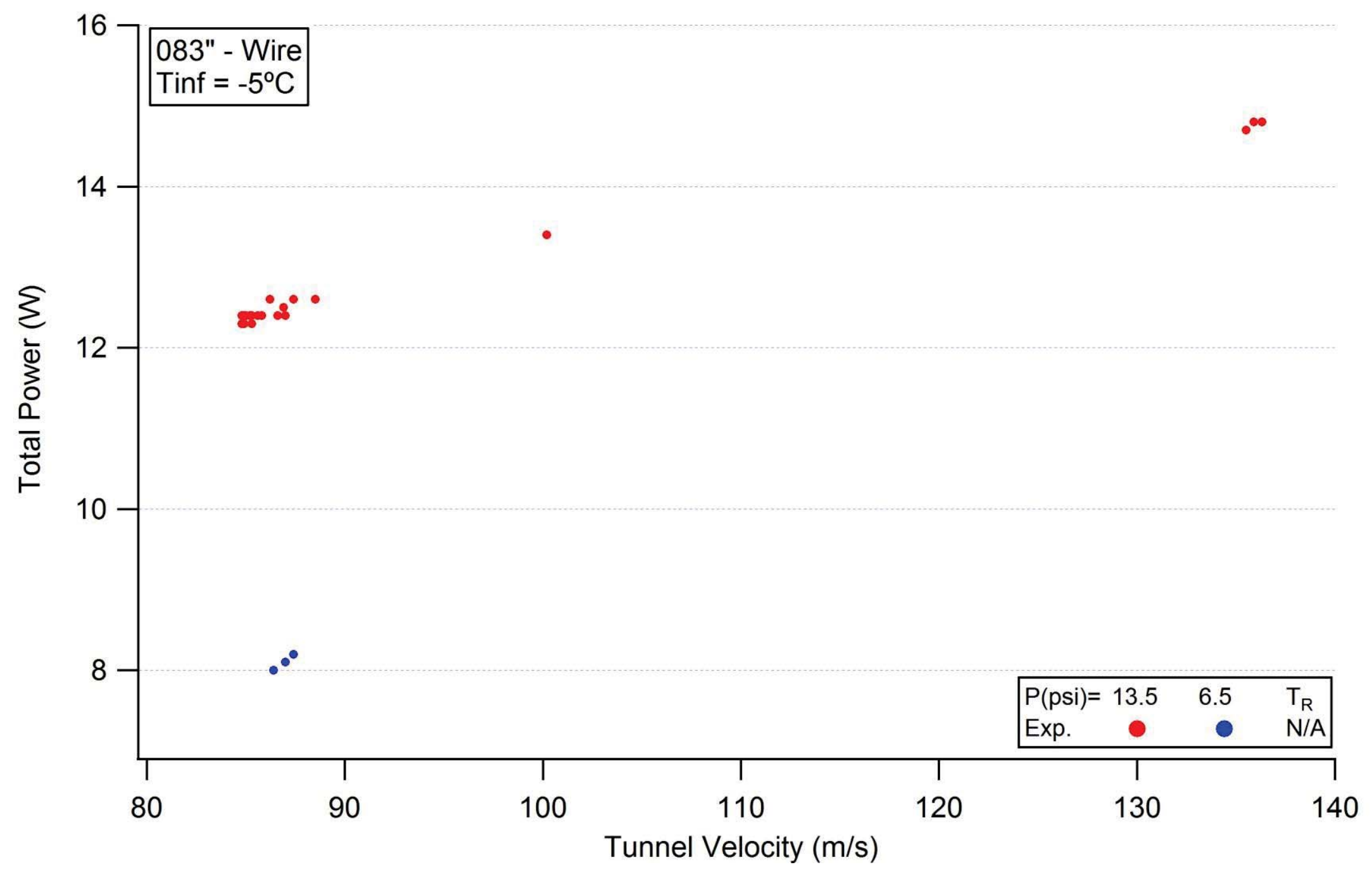




\section{Model vs. Experiment - Dry}

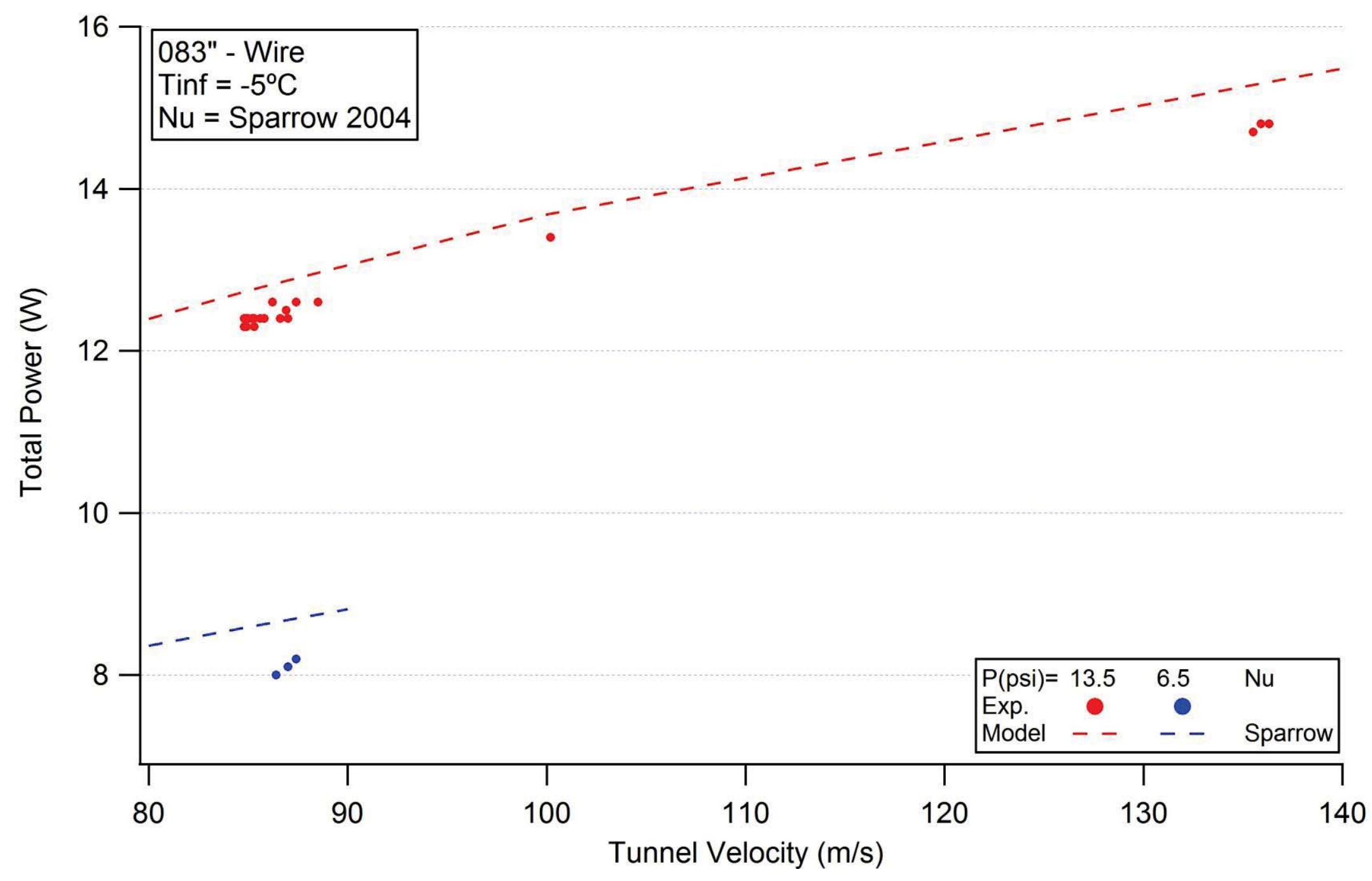




\section{Model vs. Experiment - Dry}

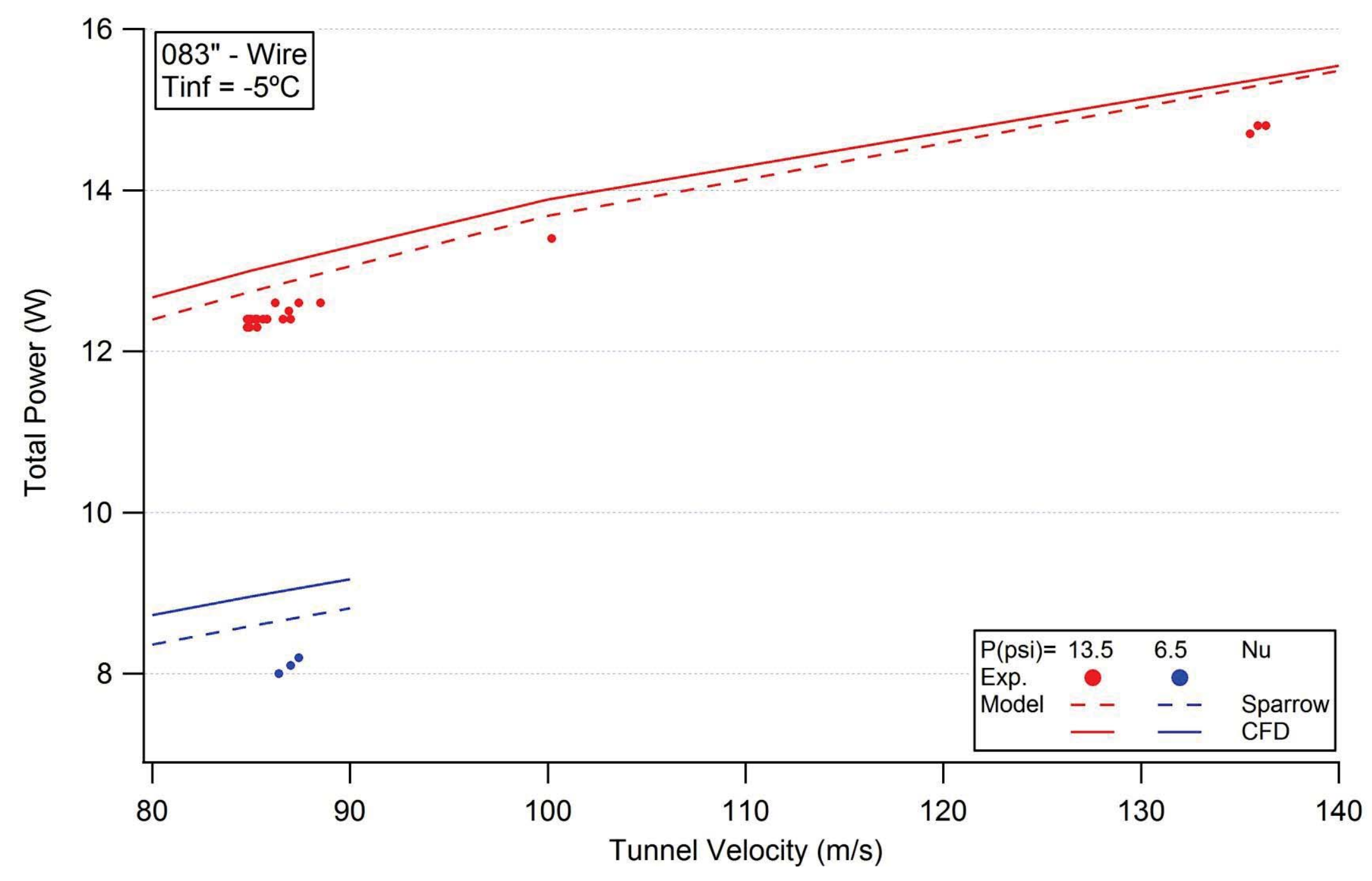




\section{Heating \& Evaporation Model}

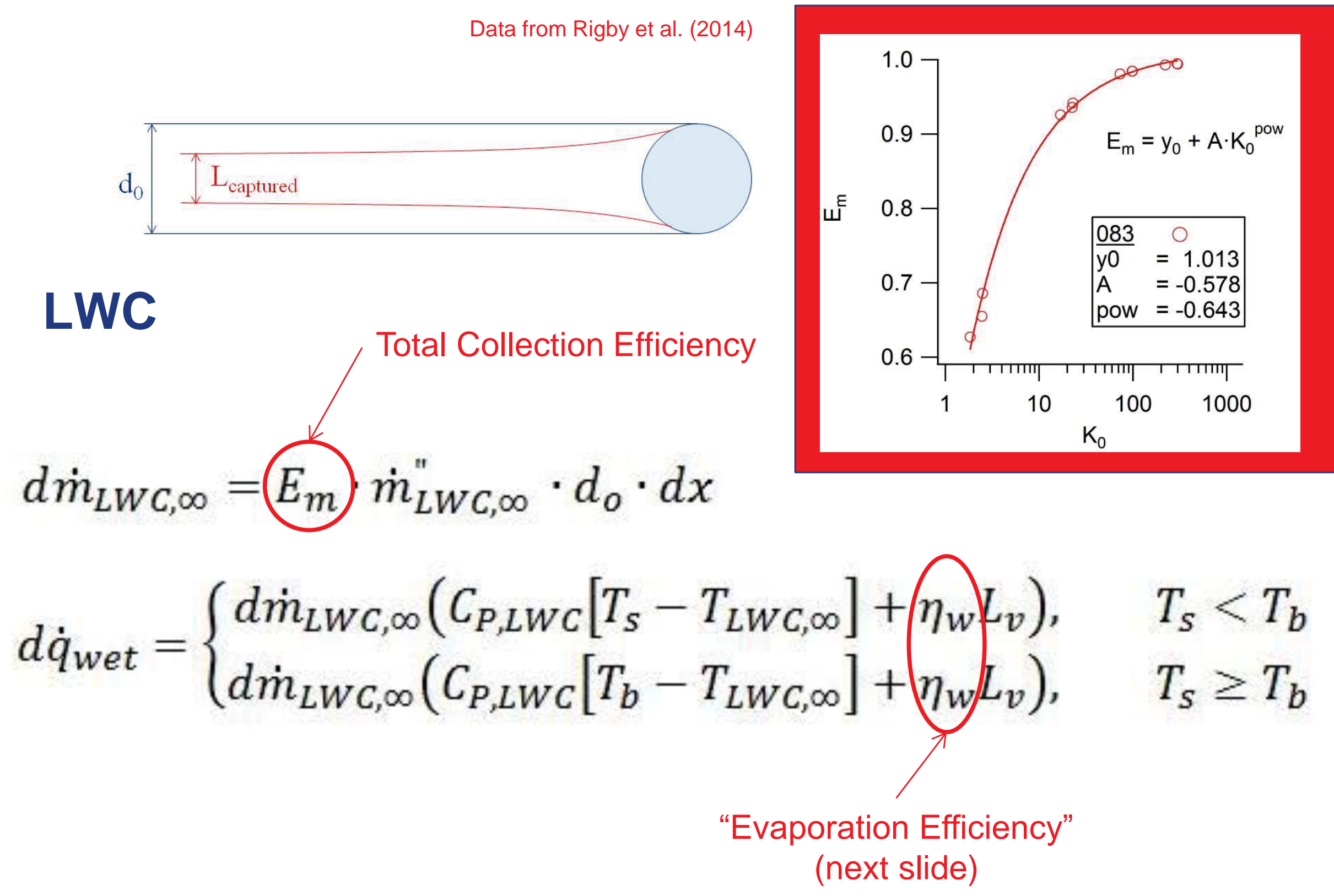




\section{Evaporation Efficiency}

- Needed a way to estimate fraction of water that does not evaporate

- Defined a parameter called evaporation efficiency, $\eta$

$$
\eta=\frac{\text { evaporation potential }}{\text { incoming mass flux }} \quad 0 \leq \eta \leq 1
$$

- Use analogy of heat \& mass transfer

- Mass flux related to heat flux

- Evaporation area:

- For 083,021 entire circumference

- For HP, forward face of element

- Does not include bounce / splash

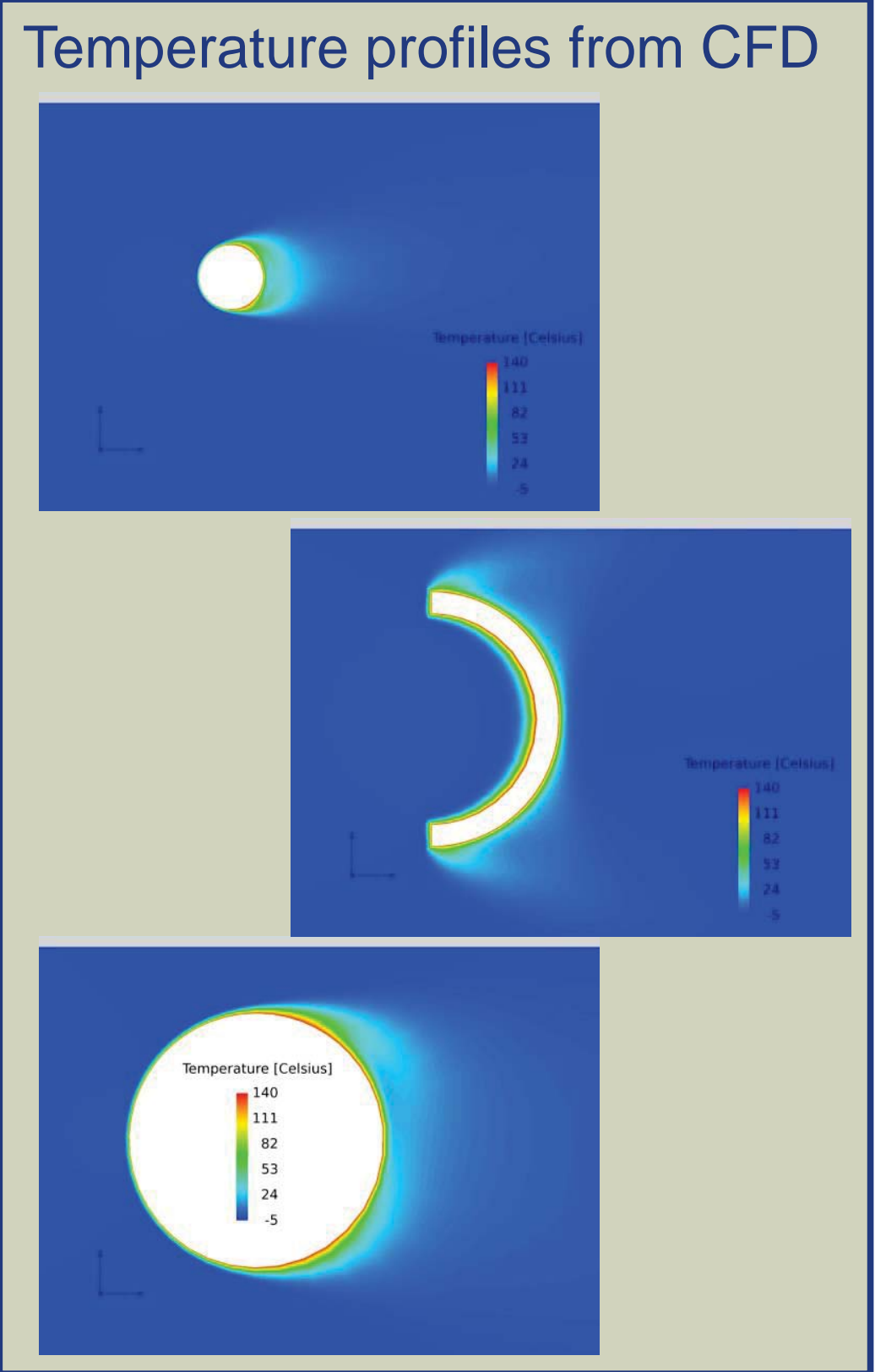




\section{Effect of LWC on Temperature Profiles}
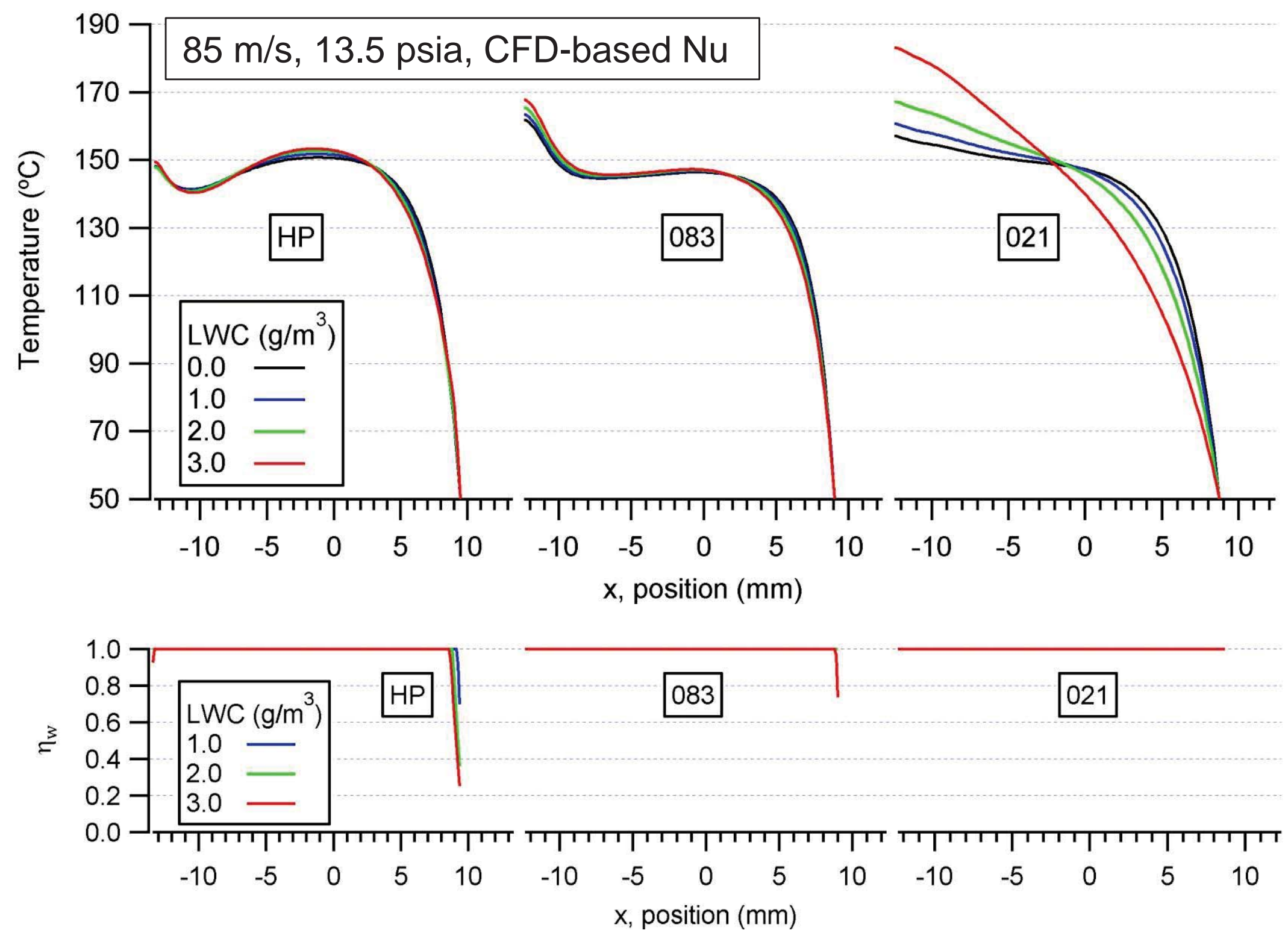


\section{Wet Power - Effect of Conduction Losses}

\begin{tabular}{|r|r|r|}
\hline Term & Dry & Wet \\
\hline LWC $\left(\mathrm{g} / \mathrm{m}^{3}\right)$ & 0 & 3 \\
\hline$\dot{q}_{\text {gen }}(\mathrm{W})$ & 6.34 & 13.61 \\
\hline$\dot{q}_{\text {conv }}(\mathrm{W})$ & -6.05 & -6.05 \\
\hline$\dot{q}_{\text {cond }}(\mathrm{W})$ & -0.28 & -0.14 \\
\hline$\dot{q}_{\text {rad }}(\mathrm{W})$ & -0.01 & -0.01 \\
\hline$\dot{q}_{\text {wet }}(\mathrm{W})$ & 0 & -7.41 \\
\hline
\end{tabular}

- Traditional wet power calculation:

$$
P_{\text {wet }}=13.61 \mathrm{~W}-6.34 \mathrm{~W}=7.27 \mathrm{~W}
$$

However, energy needed to evaporate LWC was 7.41W (1.9\% diff) Under measurement is due to conduction loss differences 
IWC
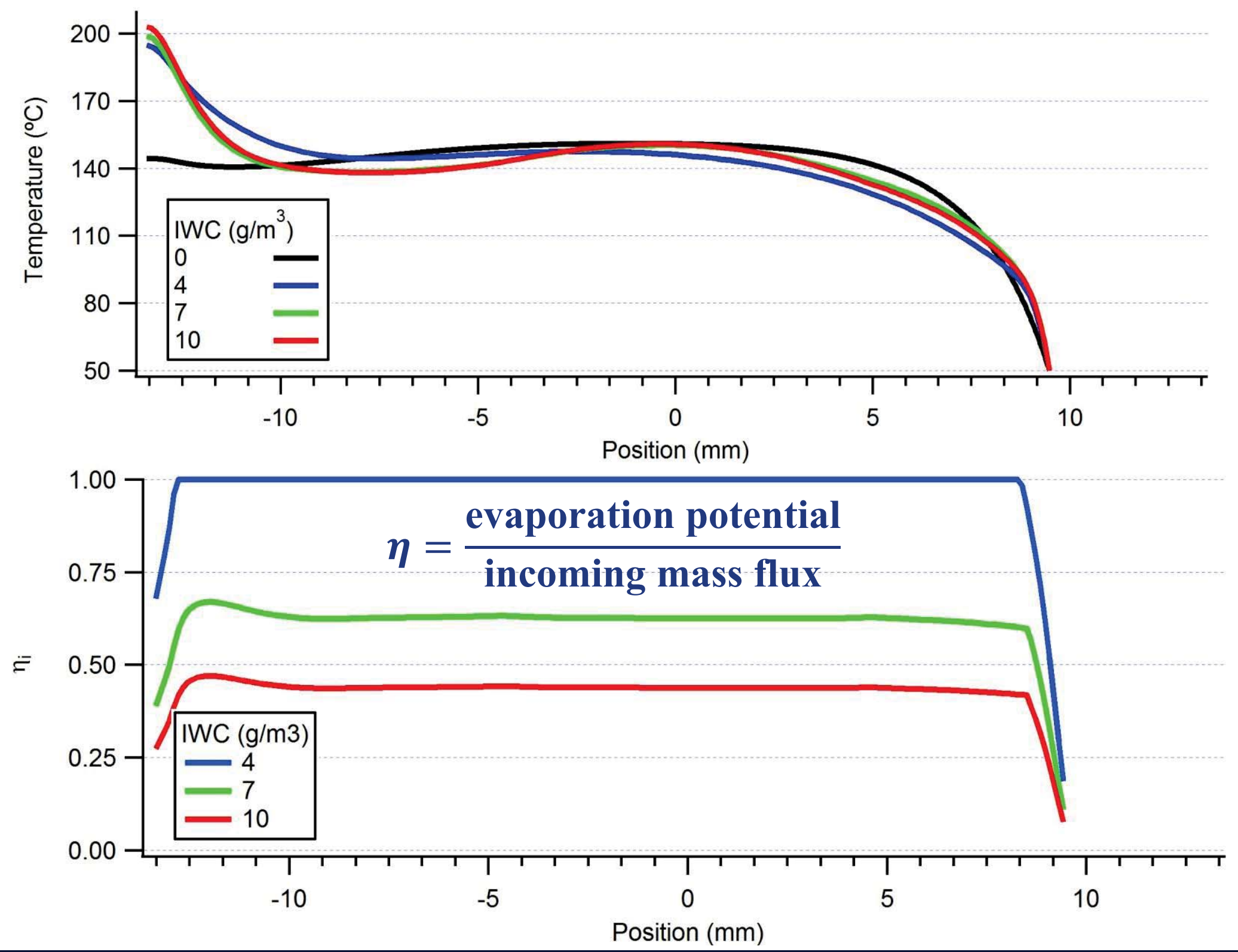


\section{Wet Power vs. IWC}

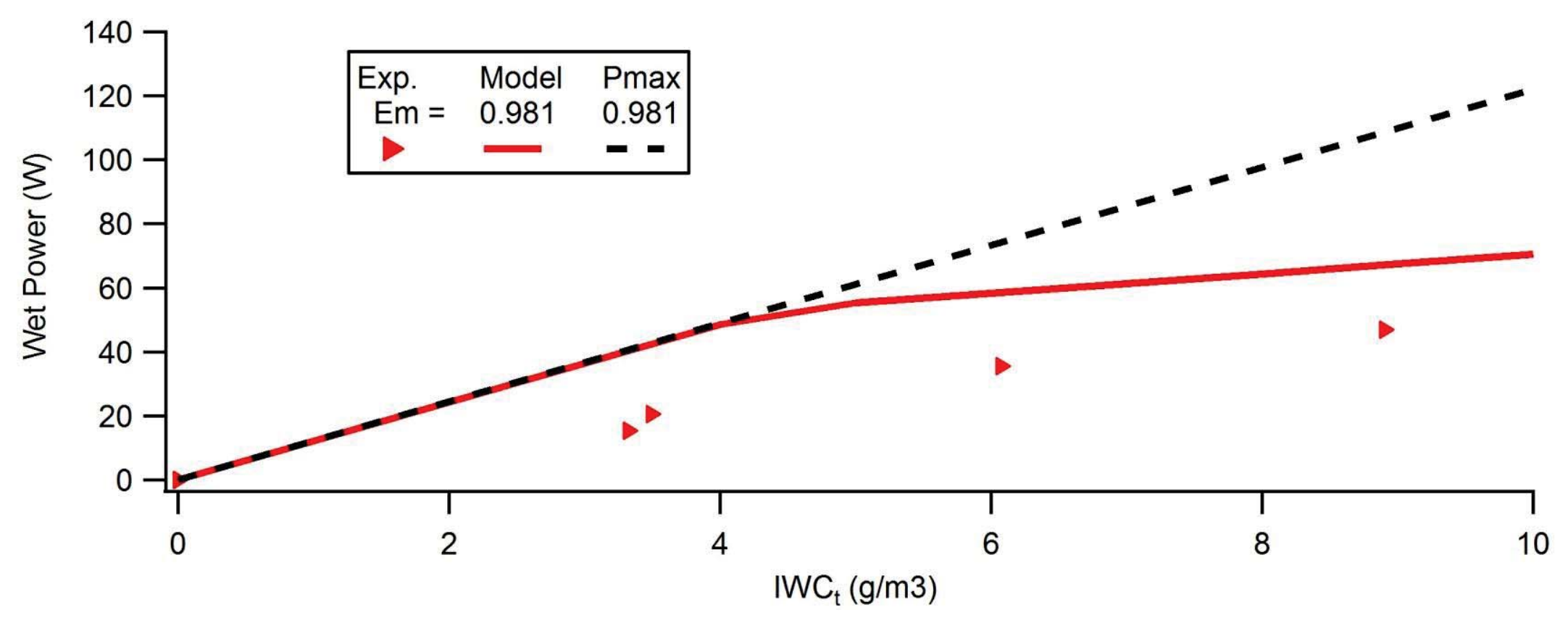




\section{Conclusions (1 of 2 )}

- Developed steady-state hot-wire thermal model

- Includes resistive heating, convection, axial conduction, radiation, and water/ice evaporation

- Examined:

- Temperature \& power variation along the wire

- Steady-state power

- Model compared to SEA multi-wire probe data

- For dry conditions:

- Matched experiment to within:

- $5.5 \%$ for 021

- $9.2 \%$ for 083

- $14 \%$ for HP

- Max. conduction loss $\sim 4 \%$ of total power for conditions examined 


\section{Conclusions (2 of 2$)$}

- Wet conditions:

- Introduced "evaporation potential" to estimate \% water evaporated

- Needs validation

- LWC:

- Affected temperature profile of 021 most significantly;

- In all cases, high evaporation potential, effect minimal

- Conduction losses can be different dry vs. wet

- For 021 at $3 \mathrm{~g} / \mathrm{m}^{3} \rightarrow 1.9 \%$ difference in $\mathrm{P}_{\text {wet }}$ measured vs. actual

- IWC (HP only)

- Model suggests a non-linear behavior of wet power and $\mathrm{IWC}_{\mathrm{t}}$

- Below $4 \mathrm{~g} / \mathrm{m}^{3}$, linear relationship

- Above $4 \mathrm{~g} / \mathrm{m}^{3}$, non linear due to incomplete evaporation everywhere along wire

- Limited available experimental data to see if trend is correct

- Bouncing or splashing-type loss present in experiment complicate interpretation

- Further examination \& development of model planned 


\section{Acknowledgments}

- This work is supported by the Atmospheric Environment Safety Technology Project (AEST) in the NASA Aviation Safety Program.

- Author acknowledgements:

- Lyle Lilie and the staff of Science Engineering Associates

- William Wright

- Colin Bidwell

- Tadas Bartkus 